\title{
Hacia una transformación digital consciente. El museo como laboratorio
}

Towards a conscious digital transformation. The museum as a laboratory

\section{Marta Font Cifré}

Responsable de Programación y Mediación. K6 Gestión Cultural. mfont@k6gestioncultural.com

\begin{abstract}
Resumen
Desde el museo quizá hasta la llegada de la pandemia Covid19 y la consiguiente declaración del estado de alarma, que alteró el funcionamiento habitual de este espacio, no ha habido una estrategia clara en torno a su dimensión digital. En estos meses el museo ha pasado por varios procesos que han puesto de manifiesto la necesidad de cuestionar sus propias prácticas en un contexto bañado por la incertidumbre y el peso de la actividad no presencial. Ante esta realidad, la necesidad de la definición de una ESTRATEGIA DIGITAL como una línea transversal del museo, se ha situado como una prioridad. Pero, ¿qué significa "estrategia digital”, ¿qué implica? Seguramente requiere revisar el propio proyecto museístico, los objetivos del museo, profundizar en el conocimiento de los públicos, definir una experiencia de persona usuaria clara, una estrategia de contenidos, los canales de comunicación, y hacerlo en términos de ACCESIBILIDAD.
\end{abstract}

Plantear qué significa actualmente accesibilidad en el museo y qué significa accesibilidad digital puede ayudar a identificar los retos que aún tiene en esta materia.

Esta reflexión se enmarca en el proyecto Diagnóstico de Accesibilidad de los Museos de Euskadi, desarrollado por K6 Gestión Cultural en 2020 por solicitud de Gobierno Vasco. Actualmente, desde el grupo que conforma el equipo de trabajo seguimos investigando en torno a la accesibilidad digital en los museos.

Palabras clave: museo digital, innovación social, accesibilidad, estrategia digital. 


\begin{abstract}
Up until the Covid-19 pandemic and the ensuing state of alarm that altered its normal functioning the museum didn't have a clear strategy regarding its digital aspects. During those months of uncertainty and growing online activity, there has been an increasing need for the museum to question the usual ways of doing. In the face of this new reality, it's clear that we need to define and implement a new and all-encompassing digital strategy.

But what does "digital strategy" mean? What are the implications?

It will probably require reviewing the museum project itself and the museum's objectives, deepening the knowledge of the public, defining a content strategy and a clear user experience, reviewing the communication channels and doing so in terms of accessibility.

Asking what museum accessibility currently means and what digital accessibility is can help the museum identify the challenges it still faces in this area.

This reflection is part of the Diagnosis of Accessibility of the Museums of the Basque Country project, which we at K6 Gestion Cultural have carried out this year at the request of the Basque Government. Currently, from the working group that makes up the team, we continue researching on the digital accessibility of museums.
\end{abstract}

Keywords: digital museum, social innovation, accessibility, digital strategy 


\section{Introducción}

(...) Durante los meses de verano de 2020, cuando los museos volvieron a abrir al público, 5 de cada 10 museos informaron una caída entre el 25 y el 75\% de los visitantes, mientras que 2 de cada 10 museos incluso informaron una caída de visitantes de más del 75\% (...).

Estos datos proceden del informe final publicado en enero de 2021 por NEMO, (Network of European Museum Organisations), a partir de las respuestas proporcionadas por 600 museos -mayoritariamente europeos- en la encuesta que realizó entre el 30 de octubre y el 29 de noviembre de $2020^{1}$.

Reflejan una realidad vivida y padecida por el sector museístico derivada de los efectos de la pandemia Covid19, la consiguiente declaración del estado de alarma ${ }^{2}$ y el proceso posterior de desescalada, con las diferentes medidas sanitarias recomendadas y las restricciones sociales instauradas.

Una realidad que trastornó sin aviso previo el día a día del museo y aceleró una transformación de sus formas de relación, precipitándole a explorar nuevas vías de producción y comunicación de sus contenidos más allá de su espacio físico. Las personas visitantes dejaron de acudir a los museos y este espacio, vacío, reaccionó volcándose en la dimensión digital, produciendo en un contexto incierto y teniendo que justificar incluso las horas de trabajo de su personal, en el mejor de los casos.

La pandemia y sus efectos pusieron en evidencia un modelo basado en una visión del museo más propia de la modernidad que de la contemporaneidad. Una visión apoyada en un sistema de producción que premia la cantidad versus la calidad, que vincula al museo más a la industria cultural, que a su papel más social como un agente de cambio. Un trasfondo ya vislumbrado y reivindicado hace años por la comunidad museística de a pie.

Recordemos la exitosa publicación de Nina Simon sobre el museo participativo publicada en $2010^{3}$, que propone nuevas fórmulas para el diseño participativo, o ya más recientemente, la propia necesidad de actualizar la definición de museo desde la comunidad internacional para reforzar su utilidad social. Una iniciativa impulsada por el ICOM (Consejo Internacional de Museos) en 2016, y que después de la falta de consenso en la primera nueva propuesta de definición de museo, a día de hoy el proceso sigue en marcha ${ }^{4}$.

A estas referencias, que dibujan un museo más centrado en la persona y su experiencia, sumamos otro factor de cambio en los últimos años que ha contribuido a potenciar la

\footnotetext{
1 Véase: https://www.ne-mo.org/(Consultar apartado de referencias para acceder al Informe).

2 Real Decreto 463/2020, de 14 de marzo, por el que se declara el estado de alarma para la gestión de la situación de crisis sanitaria ocasionada por el COVID-19. https://www.boe.es/buscar/doc.php?id=BOE-A-2020-3692

${ }^{3}$ Nina Simon: The Participatory Museum. Santa Cruz, CA: Museum 2.0. 2010.

${ }^{4}$ Entre marzo y abril de 2021 el ICOM prevé poner en marcha una segunda consulta para seguir con el proceso y llegar a un consenso más mayoritario que el anterior. Véase: https://icom.museum/es/recursos/normas-y-directrices/definicion-del-museo/ Sobre la primera consulta, véase: https://elpais.com/cultura/2019/09/07/actualidad/1567856362_291943.html
} 
dimensión social del museo. Se trata de la evolución de los departamentos de educación como espacios activos en la generación de procesos y programas propios desde una pedagogía crítica, acompañada por la progresiva organización profesional de las personas educadoras y su reivindicación como sector profesional. Unos equipos de trabajo más habituados a trabajar sobre procesos relacionales que sobre resultados, sobre lo intangible y los afectos, que sobre lo cuantitativo y material ${ }^{5}$.

Estos movimientos y procesos señalados son sólo algunos indicadores de cambio, que forman parte de la evolución del museo y sus formas de relación con el entorno. Un cambio a favor de la apertura del museo a la sociedad, comprendida desde la diversidad. El museo más social en todos sus matices sigue evolucionando y más allá de la etiqueta, palpita y se hace escuchar. Lo hace desde la presencialidad en contextos en que el museo actúa como actor local, en un rol próximo a la mediación, y lo hace a otra escala, a través de los medios de comunicación social.

Ahora bien, ¿cómo profundizar en el conocimiento y escucha de las personas usuarias en un marco digital?, ¿cómo seguir trabajando los vínculos con las diferentes comunidades a través de la dimensión digital?

Son algunas de las cuestiones que siguen surgiendo en la práctica diaria de los museos y necesitan repensarse en el marco de una estrategia transversal.

En este sentido, emerge con fuerza el museo plataforma, que genera mensajes y contenidos significativos en diferentes canales, que sitúa a la persona en el centro y busca mejorar su experiencia en un doble plano físico y digital.

\section{Objetivos}

-Plantear la necesaria evolución del museo acorde a los nuevos retos sociales, producidos y/o acelerados por los efectos de la pandemia Covid19.

-Señalar necesidades y retos del museo en una transición digital transversal. -Apuntar algunas orientaciones para contribuir a una transformación digital consciente.

-Plantear los retos aún existentes en materia de accesibilidad digital.

\section{Desarrollo de la innovación}

El museo digital ha venido para quedarse. A día de hoy, incluso se puede intuir que algunas de las acciones en el ámbito digital llevadas a cabo durante los meses de pandemia tendrán su recorrido a medio y largo plazo. Aquéllas que en un museo postpandemia contengan valor

\footnotetext{
${ }^{5}$ Para profundizar en el conocimiento sobre la evolución de la mediación cultural en España se recomienda consultar el estudio realizado por Pedagogías Invisibles, por encargo de la Fundación Daniel y Nina Carasso en 2018. Disponible en: http://www.pedagogiasinvisibles.es/proyecto/fotofija/
} 
y sean útiles para las personas, como comentaba Jordi Sellas, director del IDEAL (Centro de Artes digitales de Barcelona), permanecerán o tendrán más opciones de permanecer ${ }^{6}$.

Al fin y al cabo, la innovación social surge en un proceso de búsqueda de soluciones que contribuyan a la transformación social y al bienestar de las personas, vinculado a la tranquilidad y al sentimiento de satisfacción.

Llegados hasta aquí, y en un contexto en que la cultura ha ejercido como refugio, el museo de los cuidados, relacionado con la seguridad y el bienestar probablemente tomará fuerza en un contexto postpandemia. En este marco, urge más que nunca que el museo trace una hoja de ruta en todas sus dimensiones -también en la digital- para seguir siendo un espacio de valor social.

Pensar en digital implica un cambio transversal en la organización. Implica unir objetivos del museo e intereses de las personas, afinar mensajes y personalizar los contenidos, pensar en diferentes capas de acceso y transmisión de la información. Implica entender que lo digital no es una traslación de lo físico y que quizá esta dimensión ya no será la central. Quizá estamos hablando de una convivencia entre lo físico y digital todavía con demasiado poco recorrido para asumirse de una forma natural en el día a día del museo, habituado a generar a partir de formatos físicos, como exposiciones temporales o actividades culturales diseñadas en clave presencial. Desde esta perspectiva, aún pesa la relación de los contenidos digitales con la agenda del museo y la difusión de actividades. Falta probablemente una mayor madurez digital, para otorgar un valor propio a este contenido de forma continuada y a partir de programas estables. Otra forma de aproximarse al museo que requiere también de un comisariado, digital, y de sus propias lógicas y mecanismos para seguir evolucionando de forma crítica y social.

En cierta medida parece razonable pensar que todo museo tiene un paisaje digital esbozado, de mayor o menor precisión, de más o menos calidad, reflejo de su marca digital que, seguramente, resalta o delata su marco de posibilidades. La escala del museo, el personal técnico en plantilla o/y sus recursos, son algunos aspectos que por supuesto moldean esta marca digital.

Ahora bien, probablemente para que esta huella digital (Muñoz, 2020) madure sea necesario un ejercicio previo de definición y consenso sobre qué significa el museo digital y qué implica en función de la escala de cada institución, para avanzar hacia una transición digital integral.

En este sentido, los datos del informe de la organización NEMO, mencionada anteriormente, muestran evidencias y apuntan nuevas necesidades vinculadas a la falta de recursos, conocimientos y habilidades en materia digital.

\footnotetext{
${ }^{6}$ Véase.Webminar. Museus i món digital. Els museus en tempos de la Covid-19. https://www.youtube.com/watch?v=MbA8G9Sz6Xk\&feature=youtu.be
} 
Justamente en la cuestión planteada a los museos sobre su prioridad de apoyo en la transición digital, más del $40 \%$ que respondieron confirmaban necesitar ayuda para crear una estrategia digital. Le seguía la necesidad de apoyo en una nueva infraestructura digital $(23,2 \%)$ y la necesidad de formación del personal del museo $(18,7 \%)$.

Son evidencias que indican la necesidad de organizar los procesos y acciones digitales del museo bajo una estrategia que le acompañe hacia una transformación digital consciente, cada proyecto en su escala y dentro de sus posibilidades.

La estrategia puede ayudar a establecer prioridades, alinear acciones, ahorrar esfuerzos y a optimizar los recursos existentes. Incluso, al nacer necesariamente alineada a la misión del museo, puede contribuir a reforzarla y a focalizar objetivos desde lo digital.

Para ello, al plantearse definir una estrategia es necesario conocer previamente el punto de partida en el marco digital, y a partir de ahí construir los diferentes procesos que guiarán este recorrido. Lo explica ampliamente Conxa Rodà, especialista en este ámbito, en varios foros profesionales ${ }^{7}$.

En este camino, otro factor importante y todavía pendiente en el museo, es introducir de forma más sistematizada las herramientas y procesos para el conocimiento de las personas usuarias y su comportamiento en la dimensión digital en relación a las acciones planteadas. De no ser así, el impacto digital del museo se reduce y puede derivar, como hemos vivido en el periodo de confinamiento, a una saturación de acciones digitales sin retorno.

Se hace necesario escuchar a las personas desde todas las dimensiones, sin dejar a nadie atrás. A las personas activas en el ámbito digital, usuarias del museo, y a las que no lo son. Un doble reto, aún pendiente.

En este sentido, en un momento en que los encuentros presenciales siguen condicionados, el museo debería recuperar, por un lado, conversaciones ya iniciadas con diferentes comunidades y colectivos, interrumpidas en los meses de pandemia, y por otro, establecer nuevos diálogos en un contexto no presencial. Un aspecto pendiente y a su vez relevante, para no retroceder en conquistas sociales ya alcanzadas.

En estas claves, habla Aitziber Urtasun, responsable del Departamento de Educación Estética del Museo Oteiza (Navarra) cuando alerta de la existente brecha digital y recomienda “ (...) crear, diseñar y programar con la mirada puesta en un ángulo de $360^{\circ}$ empatizando con un entorno que convive con diferentes realidades educativas, sociales, económicas y culturales ${ }^{8 .}$

\footnotetext{
${ }^{7}$ Véase, video de la sesión. "El museo, ahora más digital_Museoa, orain digitalagoa" impartida por Conxa Rodà, en el marco de las jornadas museísticas profesionales. Sesión Abierta, 2020. Disponible en: https://www.youtube.com/watch?v=xX8IiZE_LHE

${ }^{8}$ Artículo completo disponible en el cuaderno digital "El museo sin cuerpo", publicado por K6 Gestión Cultural, en diciembre 2020. Se puede descargar en: https://www.k6gestioncultural.com/portfolio-items/cuaderno-digital-el-museo-sin-cuerpo/
} 


\section{Resultados}

El museo digital necesita una estrategia, planteada en claves de accesibilidad. La tarea no es fácil, pero el debate necesita ser abordado. Cuando estamos presenciando y promoviendo un giro que dirige la mirada hacia la persona usuaria, el derecho de acceso y participación cultural de todas las personas, se debería situar en las raíces de este movimiento, La accesibilidad es esa llave que abre e incluye para garantizar una participación social real en el museo, sea cual sea su dimensión.

Desde el grupo de trabajo creado en 2018 por K6 Gestión Cultural y Elkartu. Federación Coordinadora de Personas con Discapacidad Física de Gipuzkoa, estamos planteando reflexiones y acciones a través de varios proyectos para avanzar hacia la accesibilidad universal $^{9}$ en el ámbito cultural y específicamente museístico de Euskadi.

De nuestra investigación en estos años, surgen diferentes conclusiones que hacen referencia a carencias detectadas de tipo estructural, como la falta de un marco común desde lo público, a la todavía necesaria sensibilización y formación de las y los agentes culturales y museísticos en este ámbito, entre otros aspectos.

En la dimensión digital del museo las necesidades se acrecientan y el consenso entre profesionales de los museos de Euskadi es claro. Actualmente uno de los principales retos es asegurar la accesibilidad de los contenidos digitales ${ }^{10}$.

Para comprender e intentar minimizar estas necesidades, en un primer estadio tendríamos que plantearnos qué aspectos se vinculan a la accesibilidad digital y qué implica su desarrollo en el museo.

Ésta tiene que ver con la capacidad de acceso a la información por parte de cualquier persona independientemente de sus capacidades, de manera que pueda percibir, entender, navegar e interactuar con esta información ${ }^{11}$. Por tanto, en el contexto museístico hablamos del acceso a una información y contenidos desplegados en toda su cadena de accesibilidad, que en su dimensión digital implica a procesos, servicios, productos, actividades, soportes, canales de comunicación, entre otros.

Desde una perspectiva actual, incorporar de forma transversal la accesibilidad en la dimensión digital es un beneficio destinado a todas las personas que mejorará su experiencia de una forma completa e integral. Para ello, se hace necesario en esta convivencia físicodigital que completa la experiencia de la persona usuaria, establecer una serie de principios y criterios compartidos por todos los departamentos del museo para eliminar de forma progresiva las barreras de la comunicación. A su vez, bajo esta política transversal y

\footnotetext{
${ }^{9}$ Real Decreto Legislativo 1/2013, de 29 de noviembre, por el que se aprueba el Texto Refundido de la Ley General de derechos de las personas con discapacidad y de su inclusión social. Véase: https://www.boe.es/buscar/act.php?id=BOE-A-2013-12632

${ }^{10}$ Observación obtenida de los datos resultantes de la encuesta realizada a profesionales de 39 museos de Euskadi en 2020, en el marco del Diagnóstico de Accesibilidad de los Museos de Euskadi, encargo realizado por Gobierno Vasco a este grupo de trabajo.

${ }^{11}$ Consultar fuente en: https://www.paeria.es/pdf/guia-de-contingut-digital-accessible.pdf
} 
compartida, hay que asegurar que los soportes que contienen contenidos y los canales que los transmiten respondan a criterios de accesibilidad ${ }^{12}$.

En este marco, un ejemplo de buenas prácticas lo encontramos en el Canadian Museum for Human Rights, que responde a una política de accesibilidad desplegada de forma transversal en el espacio museístico y en su dimensión digital. Ésta se materializa desde la presencia de puntos de acceso universal a los contenidos digitales en el museo, a una aplicación móvil accesible con acceso a contenidos en diferentes formatos, una cuidada edición de las publicaciones en redes sociales con la incorporación de textos alternativos en las imágenes para personas con discapacidad visual, a la incorporación de subtítulos en audiovisuales, entre otros aspectos ${ }^{13 .}$

En este contexto, también es muy recomendable escuchar y tener en cuenta la colaboración con colectivos de personas con discapacidad desde el museo. Se trata de personas usuarias con necesidades específicas potencialmente beneficiarias de las medidas de accesibilidad implantadas y conocer de primera mano sus intereses y necesidades, tener en cuenta su grado de satisfacción y evaluar conjuntamente las diferentes actuaciones puede ser un gran apoyo para avanzar hacia la accesibilidad universal.

\section{Conclusiones}

El museo necesita tiempo y perspectiva para resignificarse en su doble dimensión. Los equipos necesitan formarse, adquirir nuevas competencias y habilidades digitales. Se necesita colaborar, establecer redes profesionales de apoyo para intercambiar experiencias. El museo necesita equivocarse y hablar de sus errores en foros profesionales. Prototipar, probar, corregir, readaptar, transferir, bajo un museo laboratorio que coopera con otros sectores para innovar. Si le damos este tiempo, y encontramos nuevas formas de medir su actividad, menos aceleradas y supeditadas a las cifras, encontrará un lugar relevante, en un contexto aún incierto y cambiante. En este marco, deberíamos situar nuestra comprensión del museo digital, como un espacio que amplía sus relatos y conecta con más personas, en un contexto de nuevas posibilidades.

Antes de la pandemia el museo ya había iniciado y desarrollado procesos de digitalización, vinculados sobre todo a dar mayor visibilidad a sus colecciones online y a las posibilidades de interacción que se abrieron con el desarrollo de la web 2.0 ${ }^{14}$. Pero seguramente lo hacía todavía centrado en su propia práctica, en la difusión de sus fondos y actividades y desde dicotomías que hoy se difuminan, como los públicos presenciales y digitales.

\footnotetext{
12 Sobre accesibilidad web, consultar: http://accesibilidadweb.dlsi.ua.es/

${ }^{13}$ Ver apartado de Accesibilidad en la página web del museo. https://humanrights.ca/visit/accessibility

${ }^{14}$ Véase: https://definicion.de/web-2-0/
} 
En un escenario de reconstrucción, el museo más social de despliega con más fuerza y potencia sus posibilidades de conexión, a través de la creación de espacios digitales de valor y de redes de colaboración más amplias. Hablemos de digitalización social. Hablemos de personas, de conocimientos y experiencias, de programas que nacen desde lo digital, y conviven con actividades en el museo, en un programa híbrido. Hablemos de servicios en clave digital, de la necesidad de un comisariado digital y de nuevas formas de transmisión de los mensajes más acordes a nuevas necesidades sociales, en el marco de una necesaria revisión de las prácticas museísticas en claves de sostenibilidad.

\section{Referencias}

AAVV. (2010). Guia de contingut digital accesible. Unitat UdLxTothom. Lleida:

Universitat de Lleida. < http://www.udl.cat/ca/serveis/seu/UdLxtothom/recursos/guies > [Consulta: 2 de febrero de 2021].

AAVV. (2020). El Museo sin cuerpo. Cuaderno digital. Donostia: K6 Gestión Cultural. $<$ https://www.k6gestioncultural.com/eu/portfolio-items/saio-irekia-koaderno-digitala $>$ [Consulta: 2 de febrero de 2021].

Generalitat de Catalunya. Departament de Cultura (2020).Museus i món digital. Els museus en tempos de la Covid-19. En YouTube:

$<$ https://www.youtube.com/watch?v=MbA8G9Sz6Xk\&feature=youtu.be $>$ [Consulta: 19 de mayo de 2020].

FONT, M. ANDRADE, N. (2020) Hacia una cultura inclusiva. Museos para todas y todos. Donostia: K6 Gestión Cultural SL.

FUNDACIÓN DANIEL Y NINA CARASSO (2019). Foto Fija: situación de la mediación cultural en el Estado español 2018-2019.Valencia: Fundación Daniel y Nina Carasso Colectivo y Pedagogías Invisibles. <https://www.fondationcarasso.org/es/arteciudadano/foto-fija-situacion-de-la-mediacion-cultural-en-el-estado-espanol-20182019>[Consulta: 8 de diciembre de 2020].

Kihlström, G. (2015). Creating a digital strategy for nonprofits. Carousel30 agency.

McRae, M. Seven awesome accessibility features at the Museum. Canadian Museum for Human Rights. <https://humanrights.ca/story/seven-awesome-accessibility-features-atthe-museum $>$ [Consulta: 22 de febrero de 2021].

NEMO. Network of European Museum Organisations. (2021) Follow-up survey on the impact of the COVID-19 pandemic on museums in Europe. Final Report. $<$ https://www.ne-mo.org/advocacy/our-advocacy-work/museums-during-covid19.html $>$ [Consulta: 15 de febrero 2021]

NÚÑEZ, V. (2014). Cómo crear una estrategia de contenidos paso a paso. $<$ https://vilmanunez.com/crear-estrategia-de-contenidos $>$ [Consulta: 20 de noviembre de 2021]. 
RODÀ, C. (2017).Visitor Journey Mapping: ponernos en la piel del visitante. Barcelona: Museu Nacional d'Art de Catalunya. < https://blog.museunacional.cat/es/visitorjourney-mapping-ponernos-en-la-piel-del-visitante>[Consulta: 3 de enero de 2020].

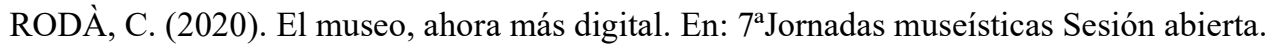
Donostia: 2020. Disponible en: <https://www.youtube.com/watch?v=xX8IiZE_LHE $>$ [Consulta: 30 de diciembre de 2020].

SANTACANA, J. (2015). La digitalización de la cultura y sus repercusiones en el museo y en el patrimonio. Museos.es. Revista de la Subdirección General de Museos Estatales Núm. 11 Pág. 82-96<https://dialnet.unirioja.es/revista/5877/A/2015>> [Consulta: 20 de febrero de 2021].

SIERRA, A. (2020) ¿Sobrevivirán los museos a la revolución digital? En YouTube: $<$ https://www.youtube.com/watch? $\mathrm{v}=6 \mathrm{pnKi6qClU} 4 \&$ feature=youtu.be $>$ [Consulta: 30 de enero de 2021].

STACK, J. (2013). Tate Digital Strategy 2013-15: Digital as a Dimension of Everything. $<$ https://www.tate.org.uk/research/publications/tate-papers/19/tate-digitalstrategy-2013-15-digital-as-a-dimension-of-everything $>$ [Consulta: 27 de diciembre de 2020]. 